\title{
MEAN VALUES AND THERMIC MAJORIZATION OF SUBTEMPERATURES
}

\author{
N.A. Watson
}

\section{Introduction}

A classical result of $F$. Riesz states that the mean values of subharmonic functions over concentric spheres of radius $r$, form convex functions of $\log r$ or $r^{2-n}$, depending on the dimension of the space [2, p. 24]. The corresponding result for subtemperatures, in which the mean values are taken over level surfaces of the Green function, was presented in [6], along with some of its consequences. In the present paper, we give a different, more elementary proof of the theorem for subtemperatures, as well as two new results on thermic majorization, one of which gives a criterion in terms of the mean values and depends upon consequences of the convexity theorem.

The principal result on thermic majorization, Theorem 3, is analogous to a well-known, elementary result on the harmonic majorization of subharmonic functions: a subharmonic function on $\mathbf{R}^{n}$ has a harmonic majorant there if and only if its mean values over all spheres centred at the origin form a bounded function. Again the mean values of a subtemperature over level surfaces of the Green function are used, and because of their geometry the whole space is replaced by a half-space $\left.\mathbf{R}^{n} \times\right]-\infty, a[$. The result requires the boundedness of means associated with a sequence of points rather than just one, and there are many technical difficulties which do not arise in the subharmonic case. For example, we have to prove that a subtemperature which has a thermic majorant on the sets $\left.\mathbf{R}^{n} \times\right]-\infty, a_{j}$ [ for all $j \in \mathbf{N}$, must also possess one on the union of those half-spaces. This result has an illuminating generalization to arbitrary open sets, which is given in Theorem 2 .

We work in $\mathbf{R}^{n+1}$, and denote a typical point by $p$ or $(x, t)$, as convenient. A particular point $p_{0}$ is assumed without comment to be $\left(x_{0}, t_{0}\right)$. A temperature is a solution of the heat equation

$$
\sum_{i=1}^{n} D_{i}^{2} u-D_{t} u=0
$$

1980 Mathematics subject classification $35 \mathrm{~K} 05,31 \mathrm{C} 05$. 
We use $\theta$ to denote the heat operator, and $\theta^{*}$ is adjoint (obtained from $\theta$ by changing the sign of $D_{t}$ ).

For all $x \in \mathbf{R}^{n}$, we put $W(x, t)=(4 \pi t)^{-n / 2} \exp \left(-\|x\|^{2} / 4 t\right)$ if $t>0$, and $W(x, t)=0$ if $t \leq 0$. Then the Green function $G$ for $\theta$ on $\mathbf{R}^{n+1}$ is given by $G(p, q)=W(x-y, t-s)$, where $p=(x, t)$ and $q=(y, s)$.

If $p_{0} \in \mathbf{R}^{n+1}$ and $c>0$, the fundamental domain $\Omega\left(p_{0}, c\right)$ is defined as $\left\{p \in \mathbf{R}^{n+1}: G\left(p_{0}, p\right)>(4 \pi c)^{-n / 2}\right\}$; it is convex and bounded. Its boundary is a smooth surface with equation $\left\|x_{0}-x\right\|=\left[2 n\left(t_{0}-t\right) \log \left(c /\left(t_{0}-t\right)\right)\right]^{1 / 2}$, together with $\left\{p_{0}\right\}$. If $\left.(x, t) \in \mathbf{R}^{n} \times\right] 0, \infty[$, we put

$$
Q(x, t)=\|x\|^{2}\left[4\|x\|^{2} t^{2}+\left(\|x\|^{2}-2 n t\right)^{2}\right]^{-1 / 2}
$$

we also put $Q(0,0)=1$. For each fixed $c>0$, the restriction to $\partial \Omega\left(p_{0}, c\right)$ of the function $(x, t) \mapsto Q\left(x_{0}-x, t_{0}-t\right)$ is continuous, and is positive except for a zero at $\left(x_{0}, t_{0}-c\right)$. If $w$ is a function on $\partial \Omega\left(p_{0}, c\right)$, we put

$$
\mathcal{M}\left(w, p_{0}, c\right)=(4 \pi c)^{-n / 2} \int_{\partial \Omega\left(p_{0}, c\right)} Q\left(x_{0}-x, t_{0}-t\right) w(x, t) d \sigma(x, t),
$$

where $\sigma$ denotes surface measure, provided that the integral exists. If $u$ is a temperature on an open set $D$, and $\bar{\Omega}\left(p_{0}, c\right) \subseteq D$, then $u\left(p_{0}\right)=\mathcal{M}\left(u, p_{0}, c\right)$. In particular, $\mathcal{M}\left(1, p_{0}, c\right)=1$ for any $p_{0}$ and $c$.

If $D$ is an open set and $p_{0} \in D$, we denote by $\Lambda\left(p_{0}\right)$ the set of all points $q \in D \backslash\left\{p_{0}\right\}$ which can be joined to $p_{0}$ by a polygonal line in $D$ along which $t$ is strictly increasing as the line is described from $q$ to $p_{0}$. A function $w$ on $D$ is called a subtemperature if it is upper semicontinuous, extended real valued but never $+\infty$, real valued on a sequence $\left\{p_{i}\right\}$ such that $D=\bigcup_{i=1}^{\infty} \Lambda\left(p_{i}\right)$, and satisfies $w\left(p_{0}\right) \leq \mathcal{M}\left(w, p_{0}, c\right)$ whenever $\bar{\Omega}\left(p_{0}, c\right) \subseteq D$. If $w$ is a subtemperature on $D$, a thermic majorant of $w$ on $D$ is a temperature $u$ such that $w \leq u$ on $D$. If $w$ has a thermic majorant on $D$, then it has a least one. The basic properties of subtemperatures are given in [4] and [5], and the equivalent class of subparabolic functions is discussed in [2].

\section{Convexity of mean values of subtemperatures}

In this section we present a more elementary proof of [6, Theorem 2] than was given in [6]. We consider subtemperatures on a domain of the form $A\left(p_{0}, c_{1}, c_{2}\right)=$ $\Omega\left(p_{0}, c_{2}\right) \backslash \bar{\Omega}\left(p_{0}, c_{1}\right)$, where $p_{0} \in \mathbf{R}^{n+1}$ and $0<c_{1}<c_{2}$. Such a domain corresponds to an annulus in the subharmonic case. We show that, if $w$ is a subtemperature on an open superset of $\bar{A}\left(p_{0}, c_{1}, c_{2}\right)$ then $\mathcal{M}\left(w, p_{0}, c\right)$ is a convex function of $c^{-n / 2}$ for $c \in\left[c_{1}, c_{2}\right]$. Our method is based on an idea due to Dinghas [1] in the subharmonic case. 
By a smooth function, we mean one for which the partial derivatives that occur in $\theta$ exist as continuous functions. For a smooth function $u$ on a domain in $\mathbf{R}^{n+1}$, we put $\nabla_{x} u=\left(D_{1} u, \ldots D_{n} u\right)$ and $\left\|\nabla_{x} u\right\|=\left(\sum_{i=1}^{n}\left(D_{i} u\right)^{2}\right)^{1 / 2}$. We use $\langle\cdot, \cdot\rangle$ to denote the inner product in $\mathbf{R}^{n}$.

It is convenient to first establish some notation and list some elementary formulas. In addition to the functions $W$ and $Q$ given above, for all $(x, t) \in$ $\left.\mathbf{R}^{n} \times\right] 0, \infty[$ we put

$$
L(x, t)=\left[4\|x\|^{2} t^{2}+\left(\|x\|^{2}-2 n t\right)^{2}\right]^{-1 / 2}
$$

and

$$
J(x, t)=2 n t \exp \left(-\|x\|^{2} / 2 n t\right) L(x, t) ;
$$

note that $Q(x, t)=\|x\|^{2} L(x, t)$. If $F \in\{W, Q, L, J\}$ and $\left(x_{0}, t_{0}\right) \in \mathbf{R}^{n+1}$, we put $F_{0}(x, t)=F\left(x_{0}-x, t_{0}-t\right)$ for all $\left.(x, t) \in \mathbf{R}^{n} \times\right]-\infty, t_{0}\left[\right.$. On $\partial \Omega\left(p_{0}, c\right)$, where

$$
\left(t_{0}-t\right) \exp \left(\left\|x_{0}-x\right\|^{2} / 2 n\left(t_{0}-t\right)\right)=c
$$

the outward unit normal $\left(\nu_{x}, \nu_{t}\right)$ is given by

$$
\nu_{x}=-2\left(t_{0}-t\right)\left(x_{0}-x\right) L_{0}(x, t), \quad \nu_{t}=\left(\left\|x_{0}-x\right\|^{2}-2 n\left(t_{0}-t\right)\right) L_{0}(x, t) .
$$

It is useful to have $\left(\nu_{x}, \nu_{t}\right)$ in terms of $J_{0}$. Since

$$
c J_{0}(x, t)=2 n\left(t_{0}-t\right)^{2} L_{0}(x, t)
$$

whenever $(x, t) \in \partial \Omega\left(p_{0}, c\right)$, we have

$$
\begin{gathered}
\nu_{x}=-\frac{c\left(x_{0}-x\right)}{n\left(t_{0}-t\right)} J_{0}(x, t), \\
\nu_{t}=\frac{c\left(\left\|x_{0}-x\right\|^{2}-2 n\left(t_{0}-t\right)\right)}{2 n\left(t_{0}-t\right)^{2}} J_{0}(x, t)
\end{gathered}
$$

for such points $(x, t)$. Next,

$$
\nabla_{x} W_{0}(x, t)=\frac{x_{0}-x}{2\left(t_{0}-t\right)} W_{0}(x, t),
$$

$$
D_{t} W_{0}(x, t)=\frac{2 n\left(t_{0}-t\right)-\left\|x_{0}-x\right\|^{2}}{4\left(t_{0}-t\right)^{2}} W_{0}(x, t),
$$


and if $g=W_{0}^{-2 / n}$ we have

$$
\theta^{*} g=\frac{2}{n}\left(\frac{2}{n}+1\right) \frac{\left\|\nabla_{x} W_{0}\right\|^{2}}{W_{0}^{2}} g
$$

since $\theta^{*} W_{0}=0$. Finally, on $\partial \Omega\left(p_{0}, c\right)$ we have

$$
-\left\langle\nabla_{x} W_{0}, \nu_{x}\right\rangle=\left\|x_{0}-x\right\|^{2} L_{0}(x, t) W_{0}(x, t)=(4 \pi c)^{-n / 2} Q_{0}(x, t) .
$$

We need certain Green identities. If $v$ and $w$ are smooth functions, it is elementary that

$$
v \theta w=\sum_{i=1}^{n} D_{i}\left(v D_{i} w\right)-\left\langle\nabla_{x} v, \nabla_{x} w\right\rangle-D_{t}(v w)+w D_{t} v
$$

and

$$
w \theta^{*} v=\sum_{i=1}^{n} D_{i}\left(w D_{i} v\right)-\left\langle\nabla_{x} w, \nabla_{x} v\right\rangle+w D_{t} v
$$

Therefore, if $A$ is any domain for which the divergence theorem is applicable,

$$
\iint_{A}\left(v \theta w+\left\langle\nabla_{x} v, \nabla_{x} w\right\rangle-w D_{t} v\right) d x d t=\int_{\partial A}\left(\left\langle v \nabla_{x} w, \nu_{x}\right\rangle-v w \nu_{t}\right) d \sigma
$$

and

$$
\iint_{A}\left(w \theta^{*} v+\left\langle\nabla_{x} w, \nabla_{x} v\right\rangle-w D_{t} v\right) d x d t=\int_{\partial A}\left\langle w \nabla_{x} v, \nu_{x}\right\rangle d \sigma
$$

Lemma 1. Let $p_{0} \in \mathbf{R}^{n+1}$, let $0<c_{1}<c_{2}$, let $u$ be a smooth function on an open superset of $\bar{A}\left(p_{0}, c_{1}, c_{2}\right)$, and put $\Omega(c)=\Omega\left(p_{0}, c\right)$ and $\mathcal{M}(c)=\mathcal{M}\left(u, p_{0}, c\right)$ for all $c \in\left[c_{1}, c_{2}\right]$. Then, if $\kappa_{n}=2^{n+1} \pi^{n / 2} n^{-1}$ and $\left.\left.c \in\right] c_{1}, c_{2}\right]$, we have

$$
\kappa_{n} c^{(n / 2)+1} \mathcal{M}_{c}(c)=\int_{\partial \Omega(c)}\left(\left\langle\nabla_{x} u, \nu_{x}\right\rangle-u \nu_{t}\right) d \sigma
$$

and

$$
\kappa_{n}\left(c^{(n / 2)+1} \mathcal{M}_{c}(c)\right)_{c}=\int_{\partial \Omega(c)} J_{0} \theta u d \sigma
$$

Proof. Let $c \in] c_{1}, c_{2}\left[\right.$, and put $A=A\left(p_{0}, c_{1}, c\right)$. We want to use (9) with this choice of $A$ and $w$ smooth, but with $v=W_{0}^{-2 / n} / 4 \pi$, so that the smoothness of 
$v$ breaks down at $p_{0}$. To prove that this is permissible, we use an approximation argument. Let $t \in] t_{0}-c_{1}, t_{0}[$, and put

$$
\begin{aligned}
F_{1}(t) & =\partial \Omega\left(p_{0}, c_{1}\right) \cap\left(\mathbf{R}^{n} \times\left[t_{0}-c_{1}, t\right]\right), \\
F_{2}(t) & =\partial \Omega\left(p_{0}, c\right) \cap\left(\mathbf{R}^{n} \times\left[t_{0}-c, t\right]\right), \\
V(t) & =A\left(p_{0}, c_{1}, c\right) \cap\left(\mathbf{R}^{n} \times\left[t_{0}-c, t\right]\right) .
\end{aligned}
$$

Applying (9) on $V(t)$ with $v=W_{0}^{-2 / n} / 4 \pi$, and using (6) and (7), we obtain

$$
\begin{gathered}
(12) \iint_{V(t)}\left(\frac{2}{n}\left(\frac{2}{n}+1\right) \frac{\left\|\nabla_{x} W_{0}\right\|^{2}}{W_{0}^{2}} v w-\frac{2 v}{n W_{0}}\left\langle\nabla_{x} w, \nabla_{x} W_{0}\right\rangle+\frac{2 v w}{n W_{0}} D_{t} W_{0}\right) d x d t \\
=\int_{\partial V(t)} \frac{-2 w v}{n W_{0}}\left\langle\nabla_{x} W_{0}, \nu_{x}\right\rangle d \sigma=\frac{2}{n}\left(c \int_{F_{2}(t)}-c_{1} \int_{F_{1}(t)}\right) w Q_{0} d \sigma .
\end{gathered}
$$

Since $w Q_{0}$ is bounded on $\partial A\left(p_{0}, c_{1}, c\right)$, as $t \rightarrow t_{0}$ - the last expression tends to

$$
\frac{2}{n}\left(c \int_{\partial \Omega(c)}-c_{1} \int_{\partial \Omega\left(c_{1}\right)}\right) w Q_{0} d \sigma
$$

For the integral over $V(t)$ in (12), the integrand is

$$
\frac{2}{n}\left(\frac{2}{n}+1\right) \frac{\left\|x_{0}-x\right\|^{2}}{4\left(t_{0}-t\right)^{2}} v w-\frac{v}{n\left(t_{0}-t\right)}\left\langle\nabla_{x} w, x_{0}-x\right\rangle+\frac{v w\left(2 n\left(t_{0}-t\right)-\left\|x_{0}-x\right\|^{2}\right)}{2 n\left(t_{0}-t\right)^{2}}
$$

by (4) and (5). Since $v, w$ and $\left\|\nabla_{x} w\right\|$ are bounded, this expression is dominated by a multiple of

$$
\frac{\left\|x_{0}-x\right\|^{2}}{\left(t_{0}-t\right)^{2}}+\frac{2 n}{\left(t_{0}-t\right)}
$$

which is obviously integrable on $V\left(t_{0}-c_{1} e^{-1}\right)$. Furthermore, in $A\left(p_{0}, c_{1}, c\right)$,

$$
\left\|x_{0}-x\right\|^{2} \geq 2 n\left(t_{0}-t\right) \log \left(c_{1} /\left(t_{0}-t\right)\right)
$$

so that on $A\left(p_{0}, c_{1}, c\right) \backslash V\left(t_{0}-c_{1} e^{-1}\right)$ we have

$$
\frac{\left\|x_{0}-x\right\|^{2}}{\left(t_{0}-t\right)^{2}} \geq \frac{2 n}{\left(t_{0}-t\right)}
$$

and therefore the expression (14) is dominated by $\left\|x_{0}-x\right\|^{2}\left(t_{0}-t\right)^{-2}$, which is integrable by [4, Lemma 4]. It follows that we can make $t \rightarrow t_{0}-$ in (12); in view of (13), we thus obtain (9) with $A=A\left(p_{0}, c_{1}, c\right)$ and $v=W_{0}^{-2 / n} / 4 \pi$. Next,

$$
\iint_{A} f d x d t=\int_{c_{1}}^{c} d \gamma \int_{\partial \Omega(\gamma)} f J_{0} d \sigma
$$


for any function $f$ such that either side exists [4, p. 388]. It therefore follows from (9) that

$\left(\int_{\partial \Omega(c)}-\int_{\partial \Omega\left(c_{1}\right)}\right) w\left\langle\nabla_{x} v, \nu_{x}\right\rangle d \sigma=\int_{c_{1}}^{c} d \gamma \int_{\partial \Omega(\gamma)}\left(w \theta^{*} v+\left\langle\nabla_{x} w, \nabla_{x} v\right\rangle-w D_{t} v\right) J_{0} d \sigma$ so that

$$
\left(\int_{\partial \Omega(c)} w\left\langle\nabla_{x} v, \nu_{x}\right\rangle d \sigma\right)_{c}=\int_{\partial \Omega(c)}\left(w \theta^{*} v+\left\langle\nabla_{x} w, \nabla_{x} v\right\rangle-w D_{t} v\right) J_{0} d \sigma
$$

In (15), we take $w=u$ and $v=W_{0}^{-2 / n} / 4 \pi$. Then, by (7),

$$
\begin{aligned}
\int_{\partial \Omega(c)} w\left\langle\nabla_{x} v, \nu_{x}\right\rangle d \sigma & =\frac{1}{4 \pi} \int_{\partial \Omega(c)} u\left(-\frac{2}{n}\right) W_{0}^{-(2 / n)-1}\left\langle\nabla_{x} W_{0}, \nu_{x}\right\rangle d \sigma \\
& =\kappa_{n} c^{(n / 2)+1} \mathcal{M}(c),
\end{aligned}
$$

so that the left side of (15) is

$$
\kappa_{n}\left(c^{(n / 2)+1} \mathcal{M}(c)\right)_{c}
$$

Next, by (6), (4), and (1),

$$
\begin{aligned}
\int_{\partial \Omega(c)} w\left(\theta^{*} v\right) J_{0} d \sigma & =\frac{1}{4 \pi} \int_{\partial \Omega(c)} u \frac{2}{n}\left(\frac{2}{n}+1\right) \frac{\left\|\nabla_{x} W_{0}\right\|^{2}}{W_{0}^{2}} W_{0}^{-2 / n} J_{0} d \sigma \\
& =\left(\frac{2}{n}+1\right) \int_{\partial \Omega(c)}\left(\frac{c\left\|x_{0}-x\right\|^{2} J_{0}}{2 n\left(t_{0}-t\right)^{2}}\right) u d \sigma \\
& =\left(\frac{2}{n}+1\right) \int_{\partial \Omega(c)} Q_{0} u d \sigma=\kappa_{n}\left(1+\frac{n}{2}\right) c^{n / 2} \mathcal{M}(c) .
\end{aligned}
$$

By (2), (3), (4) and (5), the remainder of the right side of (15) is

$$
\begin{aligned}
\frac{1}{4 \pi} \int_{\partial \Omega(c)}( & \left.-\frac{2}{n} W_{0}^{-(2 / n)-1}\left\langle\nabla_{x} u, \nabla_{x} W_{0}\right\rangle+\frac{2}{n} W_{0}^{-(2 / n)-1} u D_{t} W_{0}\right) J_{0} d \sigma \\
= & \int_{\partial \Omega(c)}\left(\left\langle\nabla_{x} u, \nu_{x}\right\rangle-u \nu_{t}\right) d \sigma .
\end{aligned}
$$

Hence (15) yields

$$
\kappa_{n}\left(c^{(n / 2)+1} \mathcal{M}(c)\right)_{c}=\kappa_{n}\left(1+\frac{n}{2}\right) c^{n / 2} \mathcal{M}(c)+\int_{\partial \Omega(c)}\left(\left\langle\nabla_{x} u, \nu_{x}\right\rangle-u \nu_{t}\right) d \sigma
$$


which implies the identity (10).

Taking $A=A\left(p_{0}, c_{1}, c\right)$ in (8), and using an argument similar to the one that gave us (15) from (9), we obtain

$$
\left(\int_{\partial \Omega(c)}\left(\left\langle v \nabla_{x} w, \nu_{x}\right\rangle-v w \nu_{t}\right) d \sigma\right)_{c}=\int_{\partial \Omega(c)}\left(v \theta w+\left\langle\nabla_{x} v, \nabla_{x} w\right\rangle-w D_{t} v\right) J_{0} d \sigma
$$

In (16), we take $v=1$ and $w=u$. Then the left side becomes

$$
\left(\int_{\partial \Omega(c)}\left(\left\langle\nabla_{x} u, \nu_{x}\right\rangle-u \nu_{t}\right) d \sigma\right)_{c}=\kappa_{n}\left(c^{(n / 2)+1} \mathcal{M}_{c}(c)\right)_{c}
$$

in view of $(10)$, and the right side becomes

$$
\int_{\partial \Omega(c)} J_{0} \theta u d \sigma
$$

thus (11) is established.

Lemma 1 provides the following elementary proof of $[4$, Theorem 12].

Corollary. Let $w$ be a subtemperature on an open set $D$, and let $p_{0} \in D$. Then $\mathcal{M}\left(w, p_{0}, \cdot\right)$ is increasing on the set of $c$ such that $\bar{\Omega}\left(p_{0}, c\right) \subseteq D$.

Proof. If $w$ is smooth on $D$, we can take $A=\Omega\left(p_{0}, c\right)$ and $v=1$ in (8), to obtain

$$
\iint_{\Omega(c)} \theta w d x d t=\int_{\partial \Omega(c)}\left(\left\langle\nabla_{x} w, \nu_{x}\right\rangle-w \nu_{t}\right) d \sigma
$$

Therefore, taking $u=w$ in (10), we obtain

$$
\mathcal{M}_{c}(c)=\kappa_{n}^{-1} c^{-(n / 2)-1} \iint_{\Omega(c)} \theta w d x d t
$$

Since $\theta w \geq 0$, this formula immediately implies that $\mathcal{M}$ is increasing. (A similar argument was given by Pini [3] for the case $n=1$.) If $w$ is an arbitrary subtemperature, we can take a decreasing sequence $\left\{w_{j}\right\}$ of smooth subtemperatures, with limit $w$ on a neighbourhood of $\bar{\Omega}\left(p_{0}, c\right)$ [2, p. 281]. Then $\mathcal{M}\left(w_{j}, p_{0}, \cdot\right)$ is increasing for every $j$, so that the same is true of its limit, which is $\mathcal{M}\left(w, p_{0}, \cdot\right)$ by the monotone convergence theorem.

We can now give a proof of [6, Theorem 2] that does not rely upon knowledge of the Dirichlet problem for $A\left(p_{0}, c_{1}, c_{2}\right)$.

Theorem 1. Let $w$ be a subtemperature on an open superset of $\bar{A}\left(p_{0}, c_{1}, c_{2}\right)$. Then there is a real-valued, convex function $\phi$ such that $\mathcal{M}\left(w, p_{0}, c\right)=\phi\left(c^{-n / 2}\right)$ for all $c \in\left[c_{1}, c_{2}\right]$. 
Proot. We require the fact that $\mathcal{M}\left(w, p_{0}, \cdot\right)$ is real-valued, which was proved in [6]. That proof requires the special case of [6, Theorem 1] in which $u$ is a temperature on an open superset of $\bar{A}\left(p_{0}, c_{1}, c_{2}\right)$, which was proved by elementary techniques (and could alternatively be deduced from (11)). It also requires the result given as an example in [6], which depends only upon the aforementioned special case of $\left[6\right.$, Theorem 1] and the fact that $\mathcal{M}\left(w, p_{0}, \cdot\right)$ is increasing (which we have just given an elementary proof of). Suppose that $w$ is smooth. Then $\theta w \geq 0$, so that

$$
\left(c^{(n / 2)+1} \mathcal{M}_{c}(c)\right)_{c} \geq 0
$$

by (11). Suppose also that $w>0$, so that $\mathcal{M}>0$. Then we can rearrange (17) to obtain

$$
\frac{\mathcal{M}_{c c}}{\mathcal{M}}+\left(\frac{n}{2 c}+\frac{1}{c}\right) \frac{\mathcal{M}_{c}}{\mathcal{M}} \geq 0
$$

Put $\lambda(c)=c^{n / 2} \mathcal{M}(c)$. Then

$$
\frac{\mathcal{M}_{c}}{\mathcal{M}}=\left(\frac{\lambda_{c}}{\lambda}-\frac{n}{2 c}\right)
$$

and

$$
\frac{\mathcal{M}_{c c}}{\mathcal{M}}=\frac{\lambda_{c c}}{\lambda}-\frac{n \lambda_{c}}{c \lambda}+\frac{n^{2}}{4 c^{2}}+\frac{n}{2 c^{2}}
$$

so that (18) becomes

$$
\frac{\lambda_{c c}}{\lambda}+\left(1-\frac{n}{2}\right) \frac{\lambda_{c}}{c \lambda} \geq 0
$$

Putting $\xi=c^{n / 2}$, we obtain

$$
\lambda_{\xi \xi}=\frac{4 c^{2-n}}{n^{2}}\left(\lambda_{c c}+\left(1-\frac{n}{2}\right) \frac{\lambda_{c}}{c}\right) \geq 0,
$$

so that $\lambda$ is a convex function of $\xi$. Hence $c^{n / 2} \mathcal{M}(c)$ is a convex function of $c^{n / 2}$, which implies that $\mathcal{M}(c)$ is a convex function of $c^{-n / 2}$.

If $w$ is smooth but not positive, we can find an open superset $S$ of $\bar{A}\left(p_{0}, c_{1}, c_{2}\right)$ and a constant $K$ such that $w-K>0$ on $S$, so that

$$
\mathcal{M}\left(w, p_{0}, c\right)=\mathcal{M}\left(w-K, p_{0}, c\right)+K
$$

is a convex function of $c^{-n / 2}$. If $w$ is not smooth, take a decreasing sequence $\left\{w_{j}\right\}$ of smooth subtemperatures that converges to $w$ on an open superset of $\bar{A}\left(p_{0}, c_{1}, c_{2}\right)[2, \mathrm{p} .281]$. Then $\left\{\mathcal{M}\left(w_{j}, p_{0}, c\right)\right\}$ is a decreasing sequence with limit $\mathcal{M}\left(w, p_{0}, c\right) \in \mathbf{R}$, so that $\mathcal{M}\left(w, p_{0}, c\right)$ is also a convex function of $c^{-n / 2}$. 
We now present a simple consequence of Theorem 1 that was not considered in [6]. The subharmonic analogue can be found in [2, p. 24].

Corollary. Let $w$ be a subtemperature on an open superset of $\bar{A}\left(p_{0}, c_{1}, c_{2}\right)$. If

$$
v(x, t)=\mathcal{M}\left(w, p_{0},\left(t_{0}-t\right) \exp \left(\left\|x_{0}-x\right\|^{2} / 2 n\left(t_{0}-t\right)\right)\right)
$$

for all $(x, t) \in A\left(p_{0}, c_{1}, c_{2}\right)$, then $v$ is a $\theta^{*}$-subtemperature (that is, a subtemperature relative to the adjoint equation).

Proof. By Theorem 1, there is a finite, convex function $\phi$ on $] c_{2}^{-n / 2}, c_{1}^{-n / 2}[$ such that $v(x, t)=\phi\left((4 \pi)^{n / 2} W\left(x_{0}-x, t_{0}-t\right)\right)$. By Lemma 1, Corollary, $\phi$ is decreasing, so that if $\psi(s)=\phi\left(c_{2}^{-n / 2}+c_{1}^{-n / 2}-s\right)$ then $\psi$ is increasing on ]$c_{2}^{-n / 2}, c_{1}^{-n / 2}\left[\right.$ and $v(x, t)=\psi\left(c_{2}^{-n / 2}+c_{1}^{-n / 2}-(4 \pi)^{n / 2} W\left(x_{0}-x, t_{0}-t\right)\right)$. Since the function of $(x, t)$ with which $\psi$ is composed to get $v$, is a solution of the adjoint equation, the dual of $\left[4\right.$, Theorem 2] implies that $v$ is a $\theta^{*}$-subtemperature.

\section{Thermic majorization}

Let $w$ be a subharmonic function on $\mathbf{R}^{n}$, and for each $r>0$ let $\mathcal{L}(w, 0, r)$ denote its mean over the sphere of radius $r$ centred at the origin. It is a wellknown, elementary result that $w$ has a harmonic majorant on $\mathbf{R}^{n}$ if and only if $\mathcal{L}(w, 0, \cdot)$ is bounded above on $] 0, \infty[$; and that, if $w$ has such a majorant and $u$ is the least one, then

$$
u(0)=\sup _{r>0} \mathcal{L}(w, 0, r)=\lim _{r \rightarrow \infty} \mathcal{L}(w, 0, r) .
$$

We seek an analogous result for subtemperatures. First, we must replace the whole space $\left(\mathbf{R}^{n+1}\right.$ in this case) by a lower half-space $\left.\mathbf{R}^{n} \times\right]-\infty, a[$, because

$$
\left.\bigcup_{c>0} \Omega\left(p_{0}, c\right)=\mathbf{R}^{n} \times\right]-\infty, t_{0}[
$$

Note that a subtemperature on $\mathbf{R}^{n+1}$ can have a thermic majorant on a half-space $\mathbf{R}^{n} \times$ ] $-\infty, 0$ [ without having one on $\mathbf{R}^{n+1}$. For example, it is well-known that there is a temperature $u$ on $\mathbf{R}^{2}$ that is identically zero on $\mathbf{R}^{n} \times$ ] $-\infty, 0$ ] but not on any open superset thereof $\left[7\right.$, p. 86]. The subtemperature $u^{+}$cannot have a thermic majorant on $\mathbf{R} \times] 0, \infty[$, since that would imply that

$$
u(x, t)=\int_{R^{n}} W(x-y, t) u(y, 0) d y=0
$$

whenever $t>0$ [7, pp. 100-102]. 
We therefore seek a necessary and sufficient condition, in terms of the surface means $\mathcal{M}$, for a subtemperature $w$ on a half-space $\left.H_{a}=\mathbf{R}^{n} \times\right]-\infty, a[$ to have a thermic majorant there. There are two essential elements for this. The first is that, if $q=(y, s) \in H_{a}$ and $\mathcal{M}(w, q, \cdot)$ is bounded above on ]0, [, then $w$ has a thermic majorant on $H_{s}$. This is far less elementary than the subharmonic theorem, and depends on the continuity of $\mathcal{M}(w, q, \cdot)$ that is implied by Theorem 1 . The second is that, if $t_{j} \rightarrow a-$ and $w$ has a thermic majorant on $H_{t_{j}}$ for every $j$, then $w$ has a thermic majorant on $H_{a}$. This result can be generalized to arbitrary open sets, and is given in Theorem 2 below.

Lemma 2. Let $w$ be a subtemperature on an open superset $D$ of $H_{a} \cup\left\{p_{a}\right\}$ for some $p_{a} \in \partial H_{a}$. If $\mathcal{M}\left(w, p_{a}, \cdot\right)$ is bounded above on $] 0, \infty[$, then there is an increasing family $\left\{w_{c}: c>0\right\}$ of subtemperatures on $D$ such that $w_{\infty}=$ $\lim _{c \rightarrow \infty} w_{c}$ is the least thermic majorant of $w$ on $H_{a}$. Furthermore,

$$
w_{\infty}\left(p_{a}\right)=\lim _{c \rightarrow \infty} \mathcal{M}\left(w, p_{a}, c\right) .
$$

Proof. If $c>0$, then there is a unique subtemperature $w_{c}$ on $D$ such that $w_{c}$ is a temperature on $\Omega\left(p_{a}, c\right), w_{c}=w$ on $D \backslash\left(\Omega\left(p_{a}, c\right) \cup\left\{p_{a}\right\}\right)$, and $w_{c} \geq w$ on $D$ [6, Theorem 5]. If $d>c$, then the same theorem yields not only $w_{d}$, but also a unique subtemperature $u_{d}$ on $D$ such that $u_{d}$ is a temperature on $\Omega\left(p_{a}, d\right)$, $u_{d}=w_{c}$ on $D \backslash\left(\Omega\left(p_{a}, d\right) \cup\left\{p_{a}\right\}\right)$, and $u_{d} \geq w_{c}$ on $D$. Since $w_{c}=w$ on a superset of $D \backslash\left(\Omega\left(p_{a}, d\right) \cup\left\{p_{a}\right\}\right)$ and $w_{c} \geq w$ on $D$, we see that $w_{d}=u_{d} \geq w_{c}$ on $D$, so that $w_{\infty}=\lim _{c \rightarrow \infty} w_{c}$ exists on $D$. By hypothesis, there is $\alpha \in \mathbf{R}$ such that $\mathcal{M}\left(w, p_{a}, c\right) \leq \alpha$ for all $c>0$. Therefore

$$
w_{c}\left(p_{a}\right) \leq \mathcal{M}\left(w_{c}, p_{a}, c\right)=\mathcal{M}\left(w, p_{a}, c\right) \leq \alpha
$$

for every $c>0$, so that $w_{\infty}\left(p_{a}\right) \leq \alpha$. If $0<c \leq d$ then, since $w_{d}$ is a subtemperature on $D$ and a temperature on $\Omega\left(p_{a}, d\right)$, we have $w_{d}\left(p_{a}\right)=\mathcal{M}\left(w_{d}, p_{a}, c\right)$ by [6, Theorem 4]. Since the mean values of subtemperatures are real-valued (by Theorem 1), we can use the monotone convergence theorem to deduce that

$$
\mathcal{M}\left(w_{\infty}, p_{a}, c\right)=\lim _{d \rightarrow \infty} \mathcal{M}\left(w_{d}, p_{a}, c\right)=w_{\infty}\left(p_{a}\right) \leq \alpha
$$

for every $c>0$. Hence $w_{\infty}$ is finite $\sigma$-a.e. on $\partial \Omega\left(p_{a}, c\right)$, and therefore the Harnack convergence theorem [2, p. 276] implies that $w_{\infty}$ is a thermic majorant of $w$ on $H_{a}$. Since any thermic majorant of $w$ on $H_{a}$ will also majorize $w_{c}$ for every $c>0$, the function $w_{\infty}$ is the least such majorant. Finally, if $c>0$ we have

$$
\mathcal{M}\left(w, p_{a}, c\right)=\mathcal{M}\left(w_{c}, p_{a}, c\right)=w_{c}\left(p_{a}\right)
$$

(by [6, Theorem 4]), from which (19) follows immediately. 
Theorem 2. Let $w$ be a subtemperature on an open set $E$, and let $\left\{p_{j}\right\}$ be a sequence in $E$ such that

$$
E=\bigcup_{j=1}^{\infty} \Lambda\left(p_{j}\right) .
$$

If $w$ has a thermic majorant on $\Lambda\left(p_{j}\right)$ for every $j$, then $w$ has a thermic majorant on $E$; and if $u$ is the least thermic majorant of $w$ on $E$, then for any $j$ the restriction of $u$ to $\Lambda\left(p_{j}\right)$ is the least thermic majorant of $w$ on $\Lambda\left(p_{j}\right)$.

Proof. Let $u_{j}$ denote the least thermic majorant of $w$ on $\Lambda\left(p_{j}\right)$. Then $u_{j}-w$ is a potential on $\Lambda\left(p_{j}\right)$, and since the Green function for $\Lambda\left(p_{j}\right)$ is the restriction to $\Lambda\left(p_{j}\right) \times \Lambda\left(p_{j}\right)$ of the Green function $G_{E}$ for $E[5 ; 2$, p. 300], we have

$$
u_{j}-w=\int_{\Lambda\left(p_{j}\right)} G_{E}(\cdot, q) d \mu_{j}(q)
$$

on $\Lambda\left(p_{j}\right)$, for some positive Borel measure $\mu_{j}$. Next, $G_{E}(p, q)>0$ if and only if $q \in \Lambda(p)[5 ; 2, \mathrm{p} .300]$, so that

$$
u_{j}(p)-w(p)=\int_{\Lambda(p)} G_{E}(p, q) d \mu_{j}(q)
$$

for all $p \in \Lambda\left(p_{j}\right)$. Next, by the form of the Riesz decomposition theorem given in [5] and the uniqueness of representing measures, $\mu_{j}$ is the measure given by the distribution $-\theta\left(u_{j}-w\right)=\theta w$ on $\Lambda\left(p_{j}\right)$. Therefore, whenever $\Lambda\left(p_{j}\right) \cap \Lambda\left(p_{k}\right) \neq \emptyset$, the measures $\mu_{j}$ and $\mu_{k}$ coincide there. In view of (20), we can therefore define a measure $\mu$ on $E$ by putting $\mu=\mu_{j}$ on every $\Lambda\left(p_{j}\right)$. This yields the representation

$$
u_{j}(p)=w(p)+\int_{\Lambda(p)} G_{E}(p, q) d \mu(q)
$$

for quasi every $p \in \Lambda\left(p_{j}\right)$. Since the right-hand side is independent of $j$, whenever $\Lambda\left(p_{j}\right) \cap \Lambda\left(p_{k}\right) \neq \emptyset$ we have $u_{j}=u_{k}$ q.e., and hence everywhere, on that intersection. We can therefore define a temperature $u$ on $E$ by putting $u=u_{j}$ on $\Lambda\left(p_{j}\right)$. Obviously $u \geq w$ on $E$, and if $v$ is a temperature on $E$ such that $v(q)<u(q)$ for some $q \in E$, then there is $i$ such that $v(q)<u_{i}(q)$ and so $v$ does not majorize $w$ on $\Lambda\left(p_{i}\right)$.

Theorem 3. Let $w$ be a subtemperature on $\left.H_{a}=\mathbf{R}^{n} \times\right]-\infty, a[$. Then $w$ has a thermic majorant on $H_{a}$ if and only if there is a sequence $\left\{p_{j}\right\}$ in $H_{a}$ such that

$$
H_{a}=\bigcup_{j=1}^{\infty} \Lambda\left(p_{j}\right)
$$


and $\mathcal{M}\left(w, p_{j}, \cdot\right)$ is bounded above on $] 0, \infty[$ for every $j$. If $w$ has a thermic majorant on $H_{a}$ and $u$ is the least one, then

$$
u(p)=\sup _{c>0} \mathcal{M}(w, p, c)=\lim _{c \rightarrow \infty} \mathcal{M}(w, p, c)
$$

for every $p \in H_{a}$.

Proof. If there is a sequence $\left\{p_{j}\right\}$ as described, then $w$ has a thermic majorant on every $\Lambda\left(p_{j}\right)$, by Lemma 2 , so that $w$ has a thermic majorant on $H_{a}$, by Theorem 2.

Conversely, if $w$ has a thermic majorant $v$ on $H_{a}$, then for any $p \in H_{a}$ and $c>0$ we have

$$
\mathcal{M}(w, p, c) \leq \mathcal{M}(v, p, c)=v(p)<\infty .
$$

Finally, if $w$ has a least thermic majorant $u$ on $H_{a}$, and $p=(x, t) \in H_{a}$, then Theorem 2 shows that the restriction of $u$ to $H_{t}$ is the least thermic majorant of $w$ on $H_{t}$. Therefore, by Lemma 2 ,

$$
u(p)=\lim _{c \rightarrow \infty} \mathcal{M}(w, p, c),
$$

and (21) follows because $\mathcal{M}(w, p, \cdot)$ is increasing.

\section{References}

[1] Dinghas, A.: Über einige Konvexitätsätze für die Mittelwerte von subharmonischen Funktionen. - J. Math. Pures Appl. 44, 1965, 223-247.

[2] Dоов, J.L.: Classical potential theory and its probabilistic counterpart. - Springer-Verlag, New York, 1984.

[3] PINI, B.: Maggioranti e minoranti delle soluzioni delle equazioni paraboliche. - Ann. Mat. Pura Appl. 37, 1954, 249-264.

[4] Watson, N.A.: A theory of subtemperatures in several variables. - Proc. London Math. Soc. (3) 26, 1973, 385-417.

[5] Watson, N.A.: Green functions, potentials, and the Dirichlet problem for the heat equation. - Proc. London Math. Soc. (3) 33, 1976, 251-298.

[6] Watson, N.A.: A convexity theorem for local mean values of subtemperatures. - Bull. London Math. Soc. 22, 1990, 245-252.

[7] Watson, N.A.: Parabolic equations on an infinite strip. - Marcel Dekker, New York, 1989.

University of Canterbury

Department of Mathematics

Christchurch 1

New Zealand

Received 23 April 1990 